\title{
Retina tissue validation of optical coherence tomography determined outer nuclear layer loss in FTLD-tau
}

\author{
Benjamin J. Kim" ${ }^{*}$ C, Vivian Lee ${ }^{1}$, Edward B. Lee ${ }^{2,3}$, Adrienne Saludades ${ }^{1}$, John Q. Trojanowski ${ }^{3}$, \\ Joshua L. Dunaief ${ }^{1}$, Murray Grossman ${ }^{4}$ and David J. Irwin ${ }^{4}$
}

\begin{abstract}
Alzheimer's disease (AD) is associated with inner retina (nerve fiber and ganglion cell layers) thinning. In contrast, we have seen outer retina thinning driven by photoreceptor outer nuclear layer (ONL) thinning with antemortem optical coherence tomography (OCT) among patients considered to have a frontotemporal degeneration tauopathy (FTLDTau). Our objective was to determine if postmortem retinal tissue from FTLD-Tau patients demonstrates ONL loss observed antemortem on OCT. Two probable FTLD-Tau patients that were deeply phenotyped by clinical and genetic testing were imaged with OCT and followed to autopsy. Postmortem brain and retinal tissue were evaluated by a neuropathologist and ocular pathologist, respectively, masked to diagnosis. OCT findings were correlated with retinal histology. The two patients had autopsy-confirmed FTLD-Tau neuropathology and had antemortem OCT measurements showing ONL thinning $(66.9 \mu \mathrm{m}$, patient \#1; $74.9 \mu \mathrm{m}$, patient \#2) below the $95 \%$ confidence interval of normal limits (75.1-120.7 $\mu \mathrm{m}$ ) in our healthy control cohort. Postmortem, retinal tissue from both patients demonstrated loss of nuclei in the ONL, matching ONL loss visualized on antemortem OCT. Nuclei counts from each area of ONL loss ( $2-3$ nuclei per column) seen in patient eyes were below the 95\% confidence interval ( $4-8$ nuclei per column for $\mathrm{ONL}$ ) of 3 normal control retinas analyzed at the same location. Our evaluation of retinal tissue from FTLD-Tau patients confirms ONL loss seen antemortem by OCT. Continued investigation of ONL thinning as a biomarker that may distinguish FTLD-Tau from other dementias is warranted.
\end{abstract}

\section{Introduction}

Tauopathies are a class of frontotemporal lobar degeneration proteinopathies (FTLD-Tau) commonly associated with frontotemporal dementia (FTD) syndromes. Clinical distinction of FTLD-Tau from other FTLD-associated proteinopathies (i.e. FTLD-TDP, FTLD-FUS) and Alzheimer's disease (AD) is challenging [8]. Thus, non-invasive biomarkers indicative of histologic features of FTLD-Tau are urgently needed to improve diagnosis and facilitate therapeutic trials.

\footnotetext{
*Correspondence: benjamin.kim@pennmedicine.upenn.edu

${ }^{1}$ Scheie Eye Institute, Department of Ophthalmology, Perelman School of Medicine, University of Pennsylvania, Philadelphia, USA

Full list of author information is available at the end of the article
}

Optical coherence tomography (OCT) enables visualization of neuronal tissue in vivo. Many studies have found inner retina thinning (nerve fiber and ganglion cell layer) in AD vs. controls [3]. In contrast, our OCT studies revealed outer retina thinning with normal inner retina thicknesses in living FTD patients with clinical features or genetic mutations predictive of FTLD-Tau pathology $[12,13,18]$. The outer retina thinning is driven by loss of the outer nuclear layer (ONL), which consists of photoreceptor nuclei and composes most of the outer retina's thickness. This outer retina thinning correlates with global cognitive impairment, and longitudinal OCT analysis found persistent and progressive ONL thinning among the probable FTLD-Tau patients $[12,13]$.

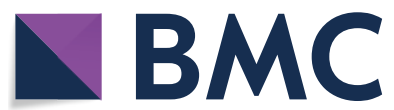

(c) The Author(s) 2021. Open Access This article is licensed under a Creative Commons Attribution 4.0 International License, which permits use, sharing, adaptation, distribution and reproduction in any medium or format, as long as you give appropriate credit to the original author(s) and the source, provide a link to the Creative Commons licence, and indicate if changes were made. The images or other third party material in this article are included in the article's Creative Commons licence, unless indicated otherwise in a credit line to the material. If material is not included in the article's Creative Commons licence and your intended use is not permitted by statutory regulation or exceeds the permitted use, you will need to obtain permission directly from the copyright holder. To view a copy of this licence, visit http://creativecommons.org/licenses/by/4.0/. The Creative Commons Public Domain Dedication waiver (http://creativeco mmons.org/publicdomain/zero/1.0/) applies to the data made available in this article, unless otherwise stated in a credit line to the data. 
Postmortem retinal tissue confirmation of antemortem OCT findings is paramount to the potential development of OCT as a biomarker, but this data is lacking in FTD. Here, we report the first 2 consecutive autopsy findings of our OCT imaged FTLD-Tau patients for important postmortem validation.

\section{Methods}

Patients were followed in a clinical research program at the Penn Frontotemporal Degeneration Center (FTDC) and Scheie Eye Institute of the University of Pennsylvania. Clinical diagnosis was established at FTDC using modern clinical criteria for FTD syndromes at weekly consensus meetings as previously described $[12,13]$. Patients had genetic testing for pathogenic mutations in MAPT (OMIM: 157140), progranulin (GRN) (OMIM:138945), C9orf72 (OMIM: 61426), and other FTD related genes based on pedigree analysis as previously described [21]. Ophthalmic evaluation included a full, dilated eye exam by a retina specialist (BJK). All patients had no history of disease that would affect retinal thickness measurements, including diabetes, retinal or optic nerve disease, high refractive error ( \pm 6.00 diopter spherical equivalent), or intraocular surgery (e.g. cataract surgery) within 90 days of the eye exam. Procedures for the OCT protocol, and neuropathological diagnosis have been previously described [12, 13, 19]. Briefly, patients were imaged with a standard spectral-domain OCT protocol using the Heidelberg Spectralis (Franklin, MA, USA). Each patient had a macular volume scan with 20 degree images, 25 raster scans, and automated real time averaging of 25 scans for each raster scan. While masked to clinical information, an analyst then segmented the retinal layers using the automated Iowa Reference Algorithm (v3.6), and segmentation errors were manually corrected $[1,6,10]$. Retinal layer thickness measurements were reported after averaging the values of the central 5 regions of the ETDRS (Early Treatment of Diabetic Retinopathy Study) grid.

Autopsies were performed at the Center for Neurodegenerative Disease Research with post-mortem intervals $<12 \mathrm{~h}$. At autopsy, eyes and brain were collected. Independent evaluation of eyes and brains was performed by an ophthalmic pathologist (VL) and neuropathologist (EBL), respectively. Eyes were fixed in 10\% formalin, processed, and paraffin embedded; five $\mu \mathrm{m}$ sections were stained with hematoxylin and eosin. The ophthalmic pathologist performed histologic retinal evaluation while masked to clinical and neuropathological diagnosis. Representative sections of the whole eye were reviewed. As the study goal was to validate ONL loss seen on OCT, the evaluation of slides was directed towards the macula, where each eye was sectioned serially at multiple levels of the macula and evaluated in a uniform fashion.

Nuclei counts for the inner nuclear layer (INL) and ONL are an accepted way to evaluate retinal tissue [2, $16,22]$. In contrast, ganglion cell layer nuclei counts are known to have wide variability among normals [7], making them difficult to compare within small groups. After identifying areas of suspected macular ONL thinning, $20-30$ columns of ONL and INL nuclei were counted at intervals of 10 microns. As measurements are affected by retinal location $[2,16,22]$, the nuclei counts for each eye were then compared to ONL and INL nuclei counts at macular areas of equivalent size, location, and distance from the optic nerve from 3 different normal controls. Controls were 3 consecutive eyes obtained from the National Disease Research Interchange (Philadelphia, PA) and were from 3 subjects with no history of eye disease that would affect the retina, diabetes, or a neurodegenerative condition.

Suspected nuclei loss, either on OCT or histopathology, was considered abnormal if the measurement was outside the $95 \%$ confidence interval $(95 \% \mathrm{CI})$ of normal limits using the normal approximation method (e.g., mean $\pm(1.96 \mathrm{X}$ standard deviation)). Normal references were calculated for OCT data using previously reported control data [12], and they were calculated for retinal tissue nuclei counts from the 3 normal control retinas, yielding a normal range consistent with published nuclei counts of normal retinas [14].

This study was approved by the University of Pennsylvania Institutional Review Board and followed the tenets of the Declaration of Helsinki. All patients gave written informed consent (with caregivers if indicated).

\section{Results}

\section{Clinical evaluation and imaging}

Patient \#1 was a 63-year-old Caucasian woman diagnosed with progressive supranuclear palsy (PSP) after 3 years of progressive L-DOPA resistant Parkinsonism, falls, and executive limitations with later emerging expressive language deficits. OCT was performed prior to this patient enrolling in a trial (NCT03068468) for PSP; she received gosuranemab (Biogen), a humanized antibody that binds $\mathrm{N}$-terminal tau. Her symptoms progressed to end-stage dementia and death at age 67. Patient \#2 was a 58-year-old Caucasian woman diagnosed with the behavioral variant of FTD (bvFTD) after 5 years of progressive cognitive and behavioral symptoms, as well as features of single-word and object knowledge later in her disease course from severe anterior temporal lobe disease. She had a pathogenic mutation in MAPT (E10+16 $\mathrm{C}>\mathrm{T}$ mutation) and declined clinically with features of 


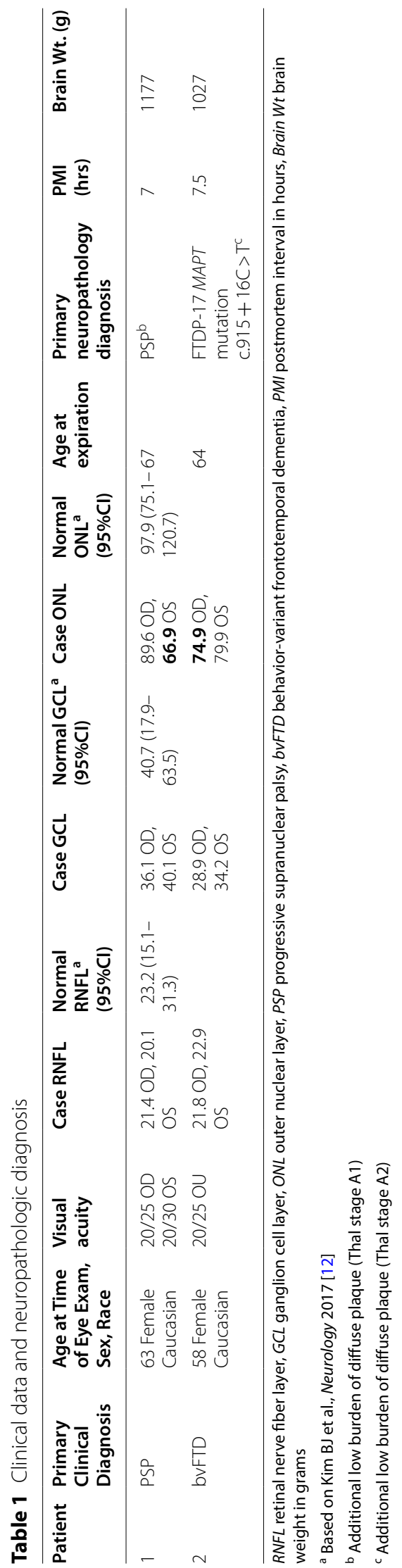


Parkinsonism and mutism with death at age 64 from endstage dementia.

Each patient's antemortem ophthalmic exam was normal and OCT images showed no known eye disease. With our imaging protocol, normal thicknesses for the retinal nerve fiber layer (RNFL), ganglion cell layer $(\mathrm{GCL})$, and outer nuclear layer (ONL) thickness are $23 \mu \mathrm{m}, 41 \mu \mathrm{m}$, and $98 \mu \mathrm{m}$, respectively [12]. As shown in Table 1, these patients had normal inner retina (RNFL and GCL) thicknesses. However, both patients had ONL thinning which was outside the $95 \%$ confidence interval thickness in our normal controls [12] $(66.9 \mu \mathrm{m}$ for left eye of patient 1 and $74.9 \mu \mathrm{m}$ for right eye of patient 2), while the contralateral eye had thinning approaching statistical significance, especially for patient \#2. With MRI, patient \#1 demonstrated asymmetric frontal lobe atrophy (Fig. 1), and patient \#2 had more widespread frontotemporal atrophy bilaterally (Fig. 2).

\section{Autopsy data}

Postmortem brain examination of patient \#1 demonstrated tau-positive threads, tufted astrocytes, coiled bodies, and perivascular vesicular astrocytes consistent with PSP with immunotherapy treatment effect (Fig. 1) [11]. Patient \#2 exhibited tau-positive pretangles, tangles, grains, and coiled bodies consistent with her known MAPT mutation (Fig. 2).

Evaluation of the retinas revealed no inner retina abnormalities. Histologically, outer retina health is commonly measured by ONL nuclei thickness. Normal maculas in the 6th-7th decade of life typically have about 6 layers of ONL nuclei [14]. For patient \#1, the right eye did not have appreciable ONL loss, but the left eye had definite ONL loss (range of 2-4 layers of nuclei) at the inferior macula, where the most ONL thinning was seen by OCT (Fig. 1). Both eyes of Patient \#2 had ONL loss (range of 2-4 layers of nuclei) within the macula corresponding to OCT findings (Fig. 2 shows left eye).

When each of these areas of ONL loss were compared to equivalent macular locations in 3 normal controls (Table 2), the ONL nuclei counts were below the 95\% confidence interval of controls (Table 3), but the INL nuclei counts of these areas were within the normal range.

\section{Discussion}

Although OCT is considered highly reproducible, there is some variability or conflicting reports on OCT findings in neurodegenerative diseases. This is contributed to, in part, by the need for gold-standard autopsy confirmation to validate the patient's specific underlying disease. This is especially true for the heterogeneous spectrum of FTLD. Therefore, tissue confirmation of OCT findings is
Table 2 Demographic data of normal control retinal tissue donors

\begin{tabular}{llllll}
\hline Control & Age (years) & Sex & Race & PMI (hrs) & Cause of Death \\
\hline 1 & 70 & Male & Caucasian & 16.5 & Cardiac Arrest \\
2 & 84 & Female & Caucasian & 4.75 & $\begin{array}{l}\text { Respiratory } \\
\text { Failure }\end{array}$ \\
3 & 85 & Male & Caucasian & 23.5 & Cardiac Arrest \\
\hline
\end{tabular}

critically important. We have presented novel and rare postmortem histopathological analysis of retinal tissue digitally quantified using OCT during life and correlated with underlying FTLD neuropathologic diagnosis. Our tissue data corroborates an outer retina abnormality seen on OCT among dementia patients. In comparison, other studies have shown retinal tissue with inner retina loss for $\mathrm{AD}$ [7] and normal OCT outer retina thickness in $\mathrm{AD}$ patients vs. controls [20].

While these patients presented with different clinical syndromes associated with tauopathies, our autopsy data aligns with our published OCT data suggesting a link between ONL thinning and FTLD-Tau, independent of clinical diagnosis [12, 13]. For each of the FTLDTau patients, significant ONL thinning was seen but there was some asymmetry with milder trends in the contralateral eye, especially for patient \#1 who also had asymmetry of cortical atrophy on MRI. Indeed, FTLD microscopic pathology and antemortem atrophy often is distributed asymmetrically in the cortex $[9,17]$ and may influence the mild laterality of OCT data in our sample here. Nonetheless, we observed significant ONL loss postmortem that corresponds to in vivo OCT data. Our observations of areas of ONL loss were supported further by an analysis of nuclei counts showing abnormally low nuclei counts within the ONL, but normal nuclei counts within the nearby INL compared to controls. The mechanisms for tau-mediated neurodegeneration affecting the ONL are unclear, but tau has been shown within human photoreceptors [15]. While the cause of ONL thinning is unknown, it may reflect phosphorylated tau toxicity related to the unusual amount of oxidative stress photoreceptors encounter [4]. Among FTLD-Tau patients, oxidative stress may promote the phosphorylation of tau within photoreceptors, which in turn may have a toxic effect on photoreceptors and explain preferential damage to photoreceptors as opposed to other neuronal cells of the retina.

The small number of cases is the primary limitation of this data and deserves emphasis. Nevertheless, we believe our rare brain and eye autopsy data from these consecutive cases is compelling as it is entirely consistent with our prior studies suggesting ONL thinning in FTLD-Tau. There was limited availability of normal control retinal 

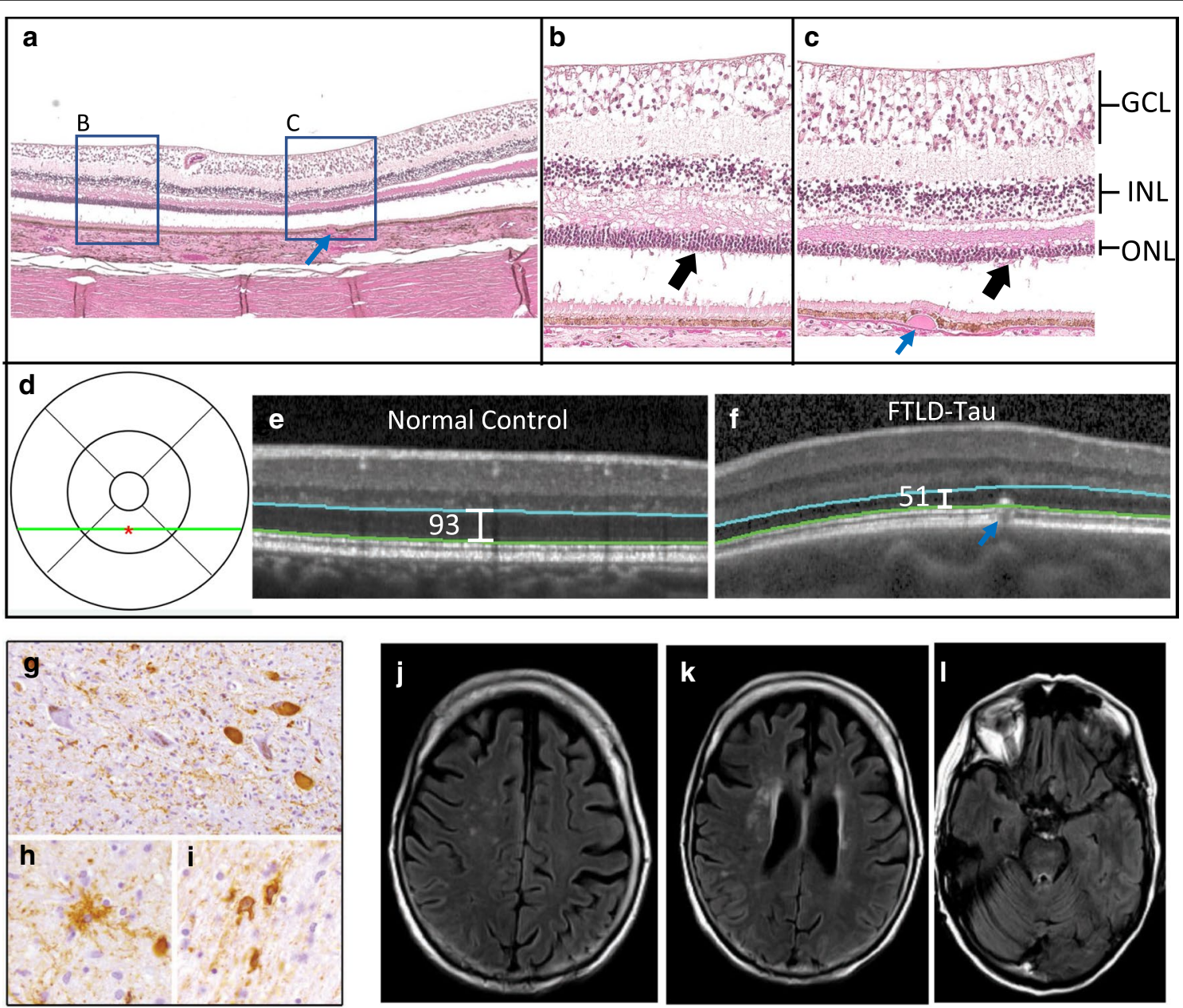

Fig. 1 Retinal tissue of patient \#1 showing outer nuclear layer (ONL) loss with spatial correlation to ONL thinning seen on optical coherence tomography (OCT) and associated brain pathology. Left eye retinal tissue from inferior parafoveal macula (a) (H \& E, original magnification X 4). This patient had a normal macula with a single druse (blue arrow) at the macula which enabled precise localization of retinal tissue. Boxes show corresponding areas of higher magnification (b, c) (original magnification X 20). This patient has some normal appearing ONL (area $\mathbf{b}$, black arrow) (about 6 layers of nuclei), but area c shows an area of ONL loss (black arrow) (2-4 layers of nuclei). Other retinal layers have no abnormalities, except for typical tissue processing artifactual separation of photoreceptor segments from the ONL and partial separation of INL from the outer plexiform layer. There is no artifact affecting ONL nuclei. Full retina examination prior to expiration showed a normal appearing macula with no confounding retinal disease. OCT at that time also showed macula ONL thinning, especially at inferior macula. $\mathbf{d}$ shows the ETDRS grid location of inferior parafoveal OCT scans (e, f) (green line) and of point measurements (red asterisk). e shows OCT of an age, sex, race matched normal control with normal ONL thickness in comparison to ONL thinning seen on this patient's OCT in which the same druse (blue arrow) seen in retinal tissue is visualized (f). OCT image ONL boundaries are shown in blue and green; Heidelberg Spectralis (Franklin, MA) caliper measurements are shown. Brain pathology showed four-repeat (4R) tauopathy with threads $(\mathbf{g})$, tufted astrocytes $(\mathbf{h})$, tangles $(\mathbf{g})$ and white matter coiled bodies within oligodendrocytes (i) consistent with progressive supranuclear palsy. MRI (j, $\mathbf{k}, \mathbf{l})$ showed prominent frontal lobe atrophy that was asymmetric and most prominent in left medial and dorsolateral frontal lobes. GCL ganglion cell layer, INL inner nuclear layer, ONL outer nuclear layer

tissue, and the eyes were from subjects of greater age than the FTLD-Tau patients. However, these subjects had nuclei counts within the expected normal range, and age is associated with mild ONL loss (not an increase) [5], providing a fair comparison to our patients to support the finding of significant ONL loss. We also acknowledge that patient \#1 received anti-tau immunotherapy with unclear effects on underlying biology and patient \#2 had hereditary tauopathy. Thus, additional autopsy samples are needed to confirm findings, test generalization for sporadic tauopathies, and contrast with clinically similar $\mathrm{AD}$ and FTLD-TDP.

To our knowledge, these data are the first human tissue confirmation of OCT abnormalities in FTD, and the first tissue confirmation of an outer retina abnormality seen in dementia patients examined and imaged 

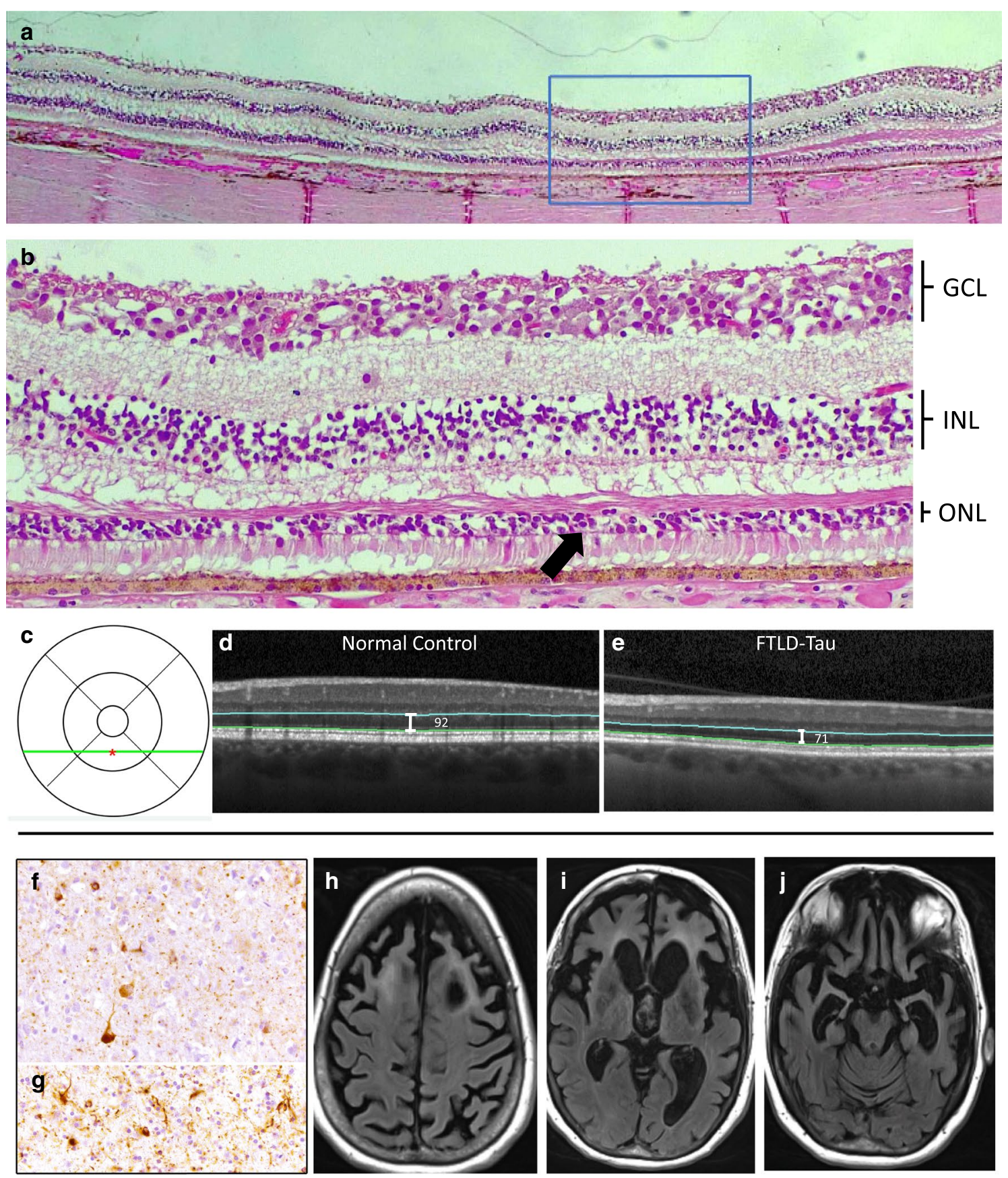

Fig. 2 Retinal tissue of patient \#2 showing outer nuclear layer (ONL) loss, correlation to ONL thinning seen on optical coherence tomography, and associated brain pathology. Left eye retina tissue from macula a ( $H$ \& E, original magnification X 5). Box shows corresponding area of higher magnification b (original magnification $\times 20$ ). There is a significant area of ONL thinning (black arrow) with only $2-4$ layers of nuclei. Other retinal layers have no abnormalities except for mild tissue processing artifact. There is no artifact affecting ONL nuclei. Full retina examination prior to expiration showed a normal appearing macula with no confounding retinal disease. OCT at that time also showed macula ONL thinning. c shows the ETDRS grid location of inferior parafoveal OCT scans $\mathbf{d}$, e (green line) and of point measurements (red asterisk). $\mathbf{d}$ shows OCT of an age, sex, race matched normal control with normal ONL thickness in comparison to ONL thinning seen on this patient's OCT (e). Brain pathology displayed tau pathology consisting of pretangles $(\mathbf{f})$, tangles $(\mathbf{f})$, grains $(\mathbf{f}, \mathbf{g})$ and white matter coiled bodies $(\mathbf{g})$ consistent with frontotemporal dementia with parkinsonism linked to chromosome 17. MRI (h, $\mathbf{i}$, j) showed severe widespread frontotemporal atrophy bilaterally. GCL ganglion cell layer, INL inner nuclear layer, ONL outer nuclear layer 
Table 3 Outer nuclear layer and inner nuclear layer nuclei counts for patient eyes and controls

\begin{tabular}{llllll}
\hline $\begin{array}{l}\text { FTLD-Tau } \\
\text { patient }\end{array}$ & Eye & $\begin{array}{l}\text { ONL nuclei count of } \\
\text { patient eye }\end{array}$ & $\begin{array}{l}\text { Average ONL nuclei count of } \mathbf{3} \\
\text { control eyes }(\mathbf{9 5 \%} \mathbf{C l})\end{array}$ & $\begin{array}{l}\text { INL nuclei count of } \\
\text { patient eye }\end{array}$ & $\begin{array}{l}\text { Average INL nuclei count } \\
\text { of } \mathbf{3} \text { control eyes } \mathbf{( 9 5 \%} \mathbf{C l})\end{array}$ \\
\hline 1 & $\mathrm{OD}^{\mathrm{a}}$ & 6.0 & $6.0^{\mathrm{a}}(3.8-8.1)$ & 3.5 & $5.0^{\mathrm{a}}(2.4-7.5)$ \\
& $\mathrm{OS}^{\mathrm{a}}$ & 3.1 & $6.0^{\mathrm{a}}(3.8-8.1)$ & 6.1 & $5.0^{\mathrm{a}}(2.4-7.5)$ \\
& $\mathrm{OD}^{\mathrm{a}}$ & 3.2 & $6.0^{\mathrm{a}}(3.8-8.1)$ & 7 & $5.0^{\mathrm{a}}(2.4-7.5)$ \\
& $\mathrm{OS}^{\mathrm{b}}$ & 2.4 & $5.9^{\mathrm{b}}(3.6-8.3)$ & 5.2 & $4.1^{\mathrm{b}}(2.3-5.9)$ \\
\hline
\end{tabular}

ONL outer nuclear layer, INL inner nuclear layer, $\mathrm{Cl}$ confidence interval

${ }^{a}$ Retinal location: $2.0 \mathrm{~mm}$ from optic nerve, inferior macula

${ }^{b}$ Retinal Location: $4.6 \mathrm{~mm}$ from optic nerve, inferior macula

with OCT during life. These data support the continued investigation of retina imaging as a biomarker that may distinguish FTLD-Tau patients from other dementias including Alzheimer's disease.

\begin{abstract}
Abbreviations
AD: Alzheimer's disease; FTD: Frontotemporal degeneration; FTLD-Tau: Frontotemporal lobar degeneration tauopathy; FTLD-TDP: Frontotemporal lobar degeneration TAR DNA binding protein 43; GCL: Ganglion cell layer; INL: Inner nuclear layer; OCT: Optical coherence tomography; ONL: Outer nuclear layer; PSP: Progressive supranuclear palsy; RNFL: Retinal nerve fiber layer.
\end{abstract}

\section{Acknowledgements}

Not applicable.

\section{Authors' contributions}

BJK drafted and revised the manuscript for content, and had major roles in the acquisition of data, study concept or design, and analysis or interpretation of data. VL revised the manuscript for content, and had major roles in the acquisition of data and the analysis or interpretation of data. EBL revised the manuscript for content and had a major role in the acquisition of data and the analysis or interpretation of data. AS revised the manuscript for content and had a major role in the acquisition of data. JQT revised the manuscript for content and had a major role role in the acquisition of data. JLD revised the manuscript for content and had major roles in the acquisition of data, study concept or design, and the analysis or interpretation of data. MG had major roles in the acquisition of data, study concept or design, and the analysis or interpretation of data. DJI had a major role in the revision of the manuscript for content and major roles in the acquisition of data, study concept or design, and analysis or interpretation of data. All authors read and approved the final manuscript.

\section{Funding}

Supported by NIH (Bethesda, MD) grants including AG017586, NS053488, AG052943, 2-P30-EY01583-26, K08-EY025742 (to V.L.), NS109260 (to D.J.I.), P01-AG066597 (to M.G.) Penn Institute on Aging, and P30-AG072979 (to E.B.L.). Funding was also provided in the form of block grants for general research purposes to the Scheie Eye Institute by Research to Prevent Blindness (New York, NY) and the Paul and Evanina Mackall Foundation Trust (Chicago, IL).

\section{Availability of data and materials}

The datasets used and/or analysed during the current study are available from the corresponding author on reasonable request.

\section{Declarations}

Ethics approval and consent to participate

This study was approved by the University of Pennsylvania Institutional Review Board (\#819894). All patients (or caregivers when appropriate) gave informed consent for this study.

\section{Consent for publication}

The cases described are of patients that have expired. Consent for publication has been given by the appropriate family member of each patient.

\section{Competing interests}

The authors declare that they have no competing interests.

\section{Author details}

${ }^{1}$ Scheie Eye Institute, Department of Ophthalmology, Perelman School of Medicine, University of Pennsylvania, Philadelphia, USA. ${ }^{2}$ Translational Neuropathology Research Laboratory, Department of Pathology and Laboratory Medicine, Perelman School of Medicine, University of Pennsylvania, Philadelphia, USA. ${ }^{3}$ Center for Neurodegenerative Disease Research, Department of Pathology and Laboratory Medicine, Perelman School of Medicine, University of Pennsylvania, Philadelphia, USA. ${ }^{4}$ Frontotemporal Lobar Degeneration Center, Department of Neurology, Perelman School of Medicine, University of Pennsylvania, Philadelphia, USA.

Received: 12 September 2021 Accepted: 6 November 2021

Published online: 18 November 2021

\section{References}

1. Abramoff MD, Garvin MK, Sonka M (2010) Retinal imaging and image analysis. IEEE Rev Biomed Eng 3:169-208

2. Baumann BH, Shu W, Song Y, Sterling J, Kozmik Z, Lakhal-Littleton S et al (2019) Liver-specific, but not retina-specific, hepcidin knockout causes retinal iron accumulation and degeneration. Am J Pathol 189(9):1814-1830

3. Chan VTT, Sun Z, Tang S, Chen LJ, Wong A, Tham CC et al (2019) Spectral domain-optical coherence tomography measurements in Alzheimer's disease: a systematic review and meta-analysis. Ophthalmology 126(4):497-510. https://doi.org/10.1016/j.ophtha.2018.08.009

4. Fridlich R, Delalande F, Jaillard C, Lu J, Poidevin L, Cronin T et al (2009) The thioredoxin-like protein rod-derived cone viability factor (RdCVFL) interacts with TAU and inhibits its phosphorylation in the retina. Mol Cell Proteom 8(6):1206-18. https://doi.org/10.1074/mcp.M800406-MCP200

5. Gartner S, Henkind P (1981) Aging and degeneration of the human macula. 1. Outer nuclear layer and photoreceptors. $\mathrm{Br} J$ Ophthalmol 65(1):23-28

6. Garvin MK, Abramoff MD, Wu X, Russell SR, Burns TL, Sonka M (2009) Automated 3-D intraretinal layer segmentation of macular spectraldomain optical coherence tomography images. IEEE Trans Med Imaging 28:1436-1447

7. Hinton DR, Sadun AA, Blanks JC, Miller CA (1986) Optic-nerve degeneration in Alzheimer's disease. N Engl J Med 315:485-487. https://doi.org/10. 1056/NEJM198608213150804

8. Irwin DJ, Cairns NJ, Grossman M, McMillan CT, Lee EB, Van Deerlin VM et al (2015) Frontotemporal lobar degeneration: defining phenotypic diversity through personalized medicine. Acta Neuropathol 129:469-491

9. Irwin DJ, McMillan CT, Xie SX, Rascovsky K, Van Deerlin VM, Coslett HB et al (2018) Asymmetry of post-mortem neuropathology in behaviouralvariant frontotemporal dementia. Brain 141:288-301 
10. Kang L, Wu X, Chen DZ, Sonka M (2006) Optimal surface segmentation in volumetric images: a graph-theoretic approach. IEEE Trans Pattern Anal Mach Intell 28:119-134

11. Kim B, Mikytuck B, Suh E, Gibbons GS, Van Deerlin VM, Vaishnavi SN et al (2021) Tau immunotherapy is associated with glial responses in FTLD-Tau. Acta Neuropathol. https://doi.org/10.1007/s00401-021-02318-y

12. Kim BJ, Irwin DJ, Song D, Daniel E, Leveque JD, Raquib AR et al (2017) Optical coherence tomography identifies outer retina thinning in frontotemporal degeneration. Neurology 89:1604-1611. https://doi.org/10. 1212/WNL.0000000000004500

13. Kim BJ, Grossman M, Song D, Saludades S, Pan W, Dominguez-Perez S et al (2019) Persistent and progressive outer retina thinning in frontotemporal degeneration. Front Neurosci 13:298

14. Kim SY, Sadda S, Pearlman J, Humayun MS, de Juan E, Melia BM et al (2002) Morphometric analysis of the macula in eyes with disciform agerelated macular degeneration. Retina 22(4):471-477

15. Leger F, Fernagut $P$, Canron M, Leoni S, Vital C, Tison F et al (2011) Protein aggregation in the aging retina. J Neuropathol Exp Neurol 70(1):63-68

16. Masri RA, Weltzien F, Purushothuman S, Lee SCS, Martin PR, Grunert U (2021) Composition of the inner nuclear layer in human retina. Invest Oph Vis Sci 62(9):22

17. Mesulam MM, Weintraub S, Rogalski EJ, Wieneke C, Geula C, Bigio EH (2014) Asymmetry and heterogeneity of Alzheimer's and frontotemporal pathology in primary progressive aphasia. Brain 137:1176-1192

18. Sun JQ, McGeehan B, Firn K, Irwin D, Grossman M, Ying GS et al (2020) Comparison of the lowa Reference Algorithm to the Heidelberg
Spectralis optical coherence tomography segmentation algorithm. J Biophotonics 13:e201960187. https://doi.org/10.1002/jbio.201960187

19. Toledo JB, Van Deerlin VM, Lee EB, Suh ER, Baek Y, Robinson JL et al (2014) A platform for discovery: The University of Pennsylvania Integrated Neurodegenerative Disease Biobank. Alzheimers Dement 10(4):477-484

20. Uchida A, Pillai JA, Bermel R, Bonner-Jackson A, Rae-Grant A, Fernandez $\mathrm{H}$ et al (2018) Outer retinal assessment using spectral-domain optical coherence tomography in patients with alzheimer's and parkinson's disease. Invest Ophthalmol Vis Sci 59:2768-2777. https://doi.org/10.1167/ iovs.17-23240

21. Wood EM, Falcone D, Suh E, Irwin DJ, Chen-Plotkin AS, Lee EB et al (2013) Development and validation of pedigree classification criteria for frontotemporal lobar degeneration. JAMA Neurol 70(11):1411-1417. https:// doi.org/10.1001/jamaneurol.2013.3956 (PMID: 24081456)

22. Zhao L, Wang C, Song D, Li Y, Song Y, Su G et al (2014) Systemic Administration of the Antioxidant/Iron Chelator alpha-Lipoic Acid Protects Against Light-Induced Photoreceptor Degeneration in the Mouse Retina. Invest Oph Vis Sci 55:5979-5988

\section{Publisher's Note}

Springer Nature remains neutral with regard to jurisdictional claims in published maps and institutional affiliations.
Ready to submit your research? Choose BMC and benefit from:

- fast, convenient online submission

- thorough peer review by experienced researchers in your field

- rapid publication on acceptance

- support for research data, including large and complex data types

- gold Open Access which fosters wider collaboration and increased citations

- maximum visibility for your research: over $100 \mathrm{M}$ website views per year

At BMC, research is always in progress.

Learn more biomedcentral.com/submissions 